\title{
Über das Verhalten von $\alpha$-Pyrrolidoncarbonsäure im tierischen Organismus.
}

\author{
Von \\ Emil Abderhalden und Rudolf Hanslian.
}

(Aus dem physiologischen Institute der Universität Halle a. S.)

(Der Redaktion zugegangen am 30. August 1912.)

Die nahen chemischen Beziehungen der Glutaminsäure zur Pyrrolidoncarbonsäure und damit zur Pyrrolidincarbonsäure machen es wahrscheinlich, das auch im Organismus des Tieres und der Pflanze diese drei Verbindungen in Wechselbeziehung treten. Als Zwischenprodukt wäre die Pyrrolidoncarbonsäure aufzufassen, die einmal durch Reduktion in Pyrrolidincarbonsäure und durch Aufspaltung in Glutaminsäure übergehen kann, wie die folgenden Formeln zeigen.

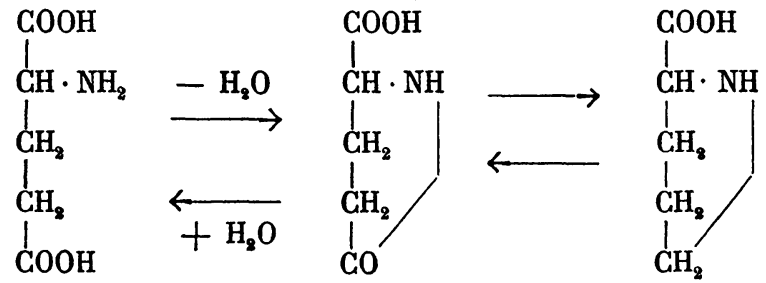

Glutaminsäure. Pyrrolidoncarbonsäure. Pyrrolidincarbonsäure.

Es sind im hiesigen Institute Untersuchungen im Gange, um zu prüfen, ob eine biologische Umwandlung von Pyrrolidoncarbonsäure in die beiden genannten Säuren stattfindet. Wir haben uns zunächst die Frage vorgelegt, ob die Pyrrolidoncarbonsäure im tierischen Organismus abgebaut wird. Die ersten Versuche wurden mit der optisch-aktiven Säure durchgeführt. Sie ergaben kein bestimmtes Resultat. Es schien, als ob die per os und auch subcutan eingeführte Säure spurlos verschwände. Wir wagten aus diesem Ergebnis keine bestimmten Schlüsse zu ziehen, weil die Möglichkeit bestand, daß die Ausscheidung der Pyrrolidoncarbonsäure eine sehr langsame ist, und sie deshalb dem Nachweis ent- 
ging. Eindeutige Resultate erhielten wir nach Verwendung von racemischer Pyrrolidoncarbonsäure. Es erschien im Harn d-Pyrrolidoncarbonsäure, woraus hervorgeht, daß die l-Komponente verändert - höchst wahrscheinlich abgebaut wurde. Damit ist bewiesen, daß der tierische Organismus und speziell das Kaninchen l-Pyrrolidoncarbonsäure verwerten kann. Bemerkt sei noch, daß Versuche am Menschen es wahrscheinlich machen, daß von diesem auch die d-Komponente in großem Umfange umgewandelt wird, wenigstens erschien im Harn keine Spur der eingeführten Säure wieder.

Zur vorläufigen Prüfung auf im Harn ausgeschiedene Pyrrolidoncarbonsäure verwendeten wir mit großem Erfolge die van Slykesche Methode. Wir bestimmten den Aminostickstoffgehalt des Harnes zunächst direkt und dann nach erfolgtem Kochen des Harnes mit Säure. Da die Pyrrolidoncarbonsäure als solche keinen Aminostickstoff enthält und dieser erst nach erfolgter Aufspaltung zu Glutaminsäure in Erscheinung tritt, wies eine erhebliche Vermehrung des Aminostickstoffgehaltes im Harn nach erfolgtem Kochen mit Säuren auf die Anwesenheit der gesuchten Säure hin.

Bemerkt sei noch, daß wir beobachtet haben, daß das Natriumsalz der Pyrrolidoncarbonsäure beim Lösen in Wasser eine ganz beträchtliche Temperaturabnahme zeigt.

Ganz indifferent scheint die Pyrrolidoncarbonsäure übrigens nicht zu sein. Nach Zufuhr von 14 und $25 \mathrm{~g}$ per os starben zwei Kaninchen. In beiden Fällen ergab die Sektion stark geblähten Dickdarm mit Entzündungserscheinungen. Beim Menschen zeigte sich starke Diarrhöe.

\section{Experimenteller Teil.}

Die zu den Versuchen verwendete dl-Pyrrolidoncarbonsäure stellten wir durch Erhitzen reiner Glutaminsäure im Ölbade auf $180-185^{\circ}$ dar. Die erhaltene reine Substanz besaß einen Schmelzpunkt von $181^{\circ}$ und eine optische Drehung gleich \pm 0 . In Berücksichtigung ihres stark sauren Geschmackes bevorzugten wir die Anwendung als Natriumsalz. 


\section{Versuch.}

$10 \mathrm{~g}$ dl-Pyrrolidoncarbonsäure wurden in $40 \mathrm{ccm}$ Wasser gelöst und mit der berechneten Menge Natriumbicarbonat $(6,5 \mathrm{~g})$ neutralisiert ${ }^{1}$ ). Nach Entfernung der Kohlensäure führten wir die ganze Lösung auf einmal einem Kaninchen mittels Schlundsonde $\mathrm{zu}$.

1. Tag.

Gesamtmenge des Harns $=210 \mathrm{ccm}$

Drehung im $10 \mathrm{~cm}-\mathrm{Rohr}=+0,1^{\circ}$

Gesamtstickstoff $\quad=0,368 \mathrm{~g}$ in $100 \mathrm{ccm}$ Harn

Aminostickstoff $\quad=0,0325 \mathrm{~g}$

Aminostickstoff nach 6 stündiger Hydrolyse mit verdünnter Schwefelsäure: $\left(40 \mathrm{ccm}\right.$ Harn $+60 \mathrm{ccm}$ verd. $\mathrm{H}_{2} \mathrm{SO}_{4}$ auf dem

Wasserbade gekocht) $=0,1045 \mathrm{~g}$ in $100 \mathrm{ccm}$ Harn Zunahme an Aminostickstoff $=0,072 \mathrm{~g}$ in $100 \mathrm{ccm}$ Harn.

\section{Tag.}

Gesamtmenge des Harns

Drehung im $10 \mathrm{~cm}-$ Rohr Gesamtstickstoff

Aminostickstoff
$=95 \mathrm{ccm}$ $= \pm 0^{\circ}$ $=1,167 \mathrm{~g}$ in $100 \mathrm{ccm}$ Harn $=0,0965 \mathrm{~g}$

$=0,0913 \mathrm{~g}$

Zunahme von Aminostickstoff $=0$.

Die Harnmenge des ersten Tages wurde auf dem Wasserbade eingedampft, der Rückstand in 70\% igem Alkohol gelöst, wiederum eingedampft und in wenig Wasser aufgenommen. Diese Lösung kochten wir mit einer überschüssigen Menge von Calciumcarbonat und filtrierten in das etwa 5 fache Volumen absoluten Alkohols. In der Kälte schieden sich nach längerem Stehen geringe Mengen eines voluminösen Niederschlags ab. Abfiltriert

1) Während das Lösen der freien Säure augenscheinlich mit keinerlei Temperaturabfall verbunden ist, zeigt sich bei der Auflösung des Natriumsalzes eine beträchtliche Temperaturabnahme. So konstatierten wir beispielsweise beim Lösen von $10 \mathrm{~g}$ Säure und $6,5 \mathrm{~g} \mathrm{NaHCO}_{3}$ in $40 \mathrm{ccm}$ Wasser von $18^{\circ}$ einen Abfall der Temperatur von $20^{\circ}$ auf $+3^{\circ}$. Wir behalten uns eine genauere Untersuchung dieser Erscheinung vor. 
und getrocknet erhielten wir $0,03 \mathrm{~g}$ Calciumsalz einer infolge Mangels an Untersuchungsmaterial nicht näher zu identifizierenden Säure.

\section{Versuch.}

Einem Kaninchen werden $28 \mathrm{~g}$ dl-pyrrolidoncarbonsaures Natrium, gelöst in physiologischer Kochsalzlösung, subcutan eingespritzt - innerhalb eines Tages viermal je $5 \mathrm{~g}$ und einmal $8 \mathrm{~g}$. Das Versuchstier befindet sich tagsüber wohlauf, nachts erfolgt der Tod.

Bei der Obduktion zeigt sich, daß an zwei Stellen die injizierte Flüssigkeit nicht resorbiert worden ist. In der Blase ist reichlich Harn vorhanden, welcher dem abgeschiedenen zugefügt wird.

Gesamtmenge Harn

Drehung im $2 \mathrm{~cm}-\mathrm{Rohr}$

Gesamtstickstoff

Aminostickstoff

$$
\begin{aligned}
& =280 \mathrm{ccm} \\
& =+0,5 \text {, } \\
& =0,812 \mathrm{~g} \text { in } 100 \mathrm{ccm} \\
& =0,03579 \text { » » } 100 \text {, } \\
& =0,203 \text { » } 100 \text { » }
\end{aligned}
$$

Aminostickstoff nach der Hydrolyse $=0,239$ \ 100 ,

Zunahme an Aminostickstoff

Der eingedampfte Harn wurde wie bei Versuch 1 weiter behandelt. Wir versuchten jedoch in diesem Falle nicht das Calcium-, sondern das Baryumsalz darzustellen. Wir kochten zu diesem Zweck die Lösung mit überschüssigem Baryumcarbonat und filtrierten in das fünffache Volumen Alkohol. In der Kälte schieden sich reichliche Mengen Baryumsalz ab. Dieses wurde im Thermostaten bei $110^{\circ}$ getrocknet und wegen seiner stark hygroskopischen Eigenschaften im Vakuumexsikkator über Phosphorpentoxyd aufbewahrt. Die Ausbeute betrug $0,9327 \mathrm{~g}$. Zu gleicher Zeit stellten wir zum Vergleich in derselben Weise das Baryumsalz der dl-Pyrrolidonsäure dar.

$0,5298 \mathrm{~g}$ der zu untersuchenden Substanz und ferner $1,6443 \mathrm{~g}$ des pyrrolidoncarbonsauren Baryums wurden in wässeriger Lösung mit der berechneten Menge 1/10-n-Schwefelsäure in der Kälte versetzt. Nach Absitzen des Niederschlags wurde die überstehende klare Flüssigkeit je mit 1 Tropfen $1 / 10-n-\mathrm{H}_{8} \mathrm{SO}_{4}$ auf Baryum geprüft. Die Reaktion war negativ. Das abfiltrierte. 
Baryumsulfat wurde getrocknet, das Filter für sich verascht und das Ganze im Platintiegel bis zur Konstanz geglüht.

$1,6443 \mathrm{~g}$ Baryumpyrrolidonat gaben $0,9766 \mathrm{~g} \mathrm{BaSO}_{4}$

$$
=0,5748 \mathrm{~g} \mathrm{Ba} \text {. }
$$

Theoretischer Wert $=0,5742$ » »

$0,5298 \mathrm{~g}$ des zu untersuchenden Baryumsalzes gaben 0,3126 $\mathrm{g}$

$$
\mathrm{BaSO}_{4}=0,1844 \mathrm{~g} \mathrm{Ba} \text {. }
$$

Theoretischer Wert $=0,1850$ »

Die beiden Zahlen stimmen sowohl untereinander wie mit dem theoretischen Wert sehr gut überein. Wir glaubten uns daher zu der Annahme berechtigt, daß das isolierte Baryumsalz pyrrolidoncarbonsaures Baryum war. In diesem Falle mußte in der vom Niederschlag abfiltrierten Flüssigkeit $0,3475 \mathrm{~g}$ der freien Pyrrolidoncarbonsäure vorhanden sein. Auf diesen Wert bezogen betrug die spezifische Drehung der Lösung $[\alpha]_{20^{\circ}}^{\mathrm{D}}=+5,706^{\circ}$.

Um die freie Säure $\mathrm{zu}$ isolieren, wurde die Lösung eingedampft, in Äthylalkohol aufgenommen und mit überschüssigem Äther gefällt. Bei längerem Stehen in Eissalzgemisch schied sich eine ölartige Schicht $a b$, welche allmählich, vorsichtig mit geringen Mengen Methylalkohol verrieben, krystallinisch erstarrte. Die Substanz besaß die spezifische Drehung $[\alpha]_{20^{0}}^{\mathrm{D}}=+8,57^{\circ}$.

Ihr Schmelzpunkt lag bei $162^{\circ}$. Der Stickstoffgehalt, nach Kjeldahl bestimmt, betrug im Durchschnitt 10,78\%, der theoretische Wert berechnet sich auf 10,85\%.

$0,1935 \mathrm{~g}$ verbrauchen $50,00 \mathrm{ccm}$

$$
\frac{-14,9 \text {. }}{35,1 \mathrm{ccm}} 1 / 10-\mathrm{n}-\mathrm{H}_{2} \mathrm{SO}_{4}=10,79 \%
$$

$0,2036 \mathrm{~g}$ verbrauchen $50,00 \mathrm{ccm}$

$$
\frac{-15,65}{34,35 \mathrm{ccm}} 1 / 10-\mathrm{n}_{2} \mathrm{H}_{2} \mathrm{SO}_{4}=10,77 \%
$$

Es war somit nach Zufuhr von dl-Pyrrolidoncarbonsäure optisch nicht ganz reine d-Pyrrolidoncarbonsäure zur Ausscheidung gelangt. 